\title{
Artículos
}

\section{Los terremotos de enero y febrero Resultados y análisis en base a la red de acelerógrafos de la UCA}

\author{
José Cepedal \\ Walter Salazar
}

\section{Resumen}

La Universidad Centroamericana "José Simeón Cañas" busca aportar con este artículo información técnica en las áreas sismológicas y de ingeniería sísmica para entender mejor los sismos principales ocurridos en El Salvador, en el período de enero y febrero del 2001. Específicamente se presentan y estudian los sismos del 13 de enero y del 13 y 17 febrero del 2001, pues son los que mayor impacto han tenido en toda la serie de eventos de estos dos meses. Así, se explican brevemente conceptos básicos de sismología, necesarios para abordar aspectos en apartados posteriores; se realiza una reseña de la sismicidad y tectónica de El Salvador y se presentan los parámetros de origen de los sismos estudiados. Posteriormente, se presentan los resultados obtenidos en la red de acelerógrafos de la UCA y un análisis breve de los registros obtenidos durante estos tres eventos. Finalmente, se hace una lista, a manera de conclusión, de algunos puntos que pueden ser beneficiosos para desarrollar la mitigación del riesgo sísmico.

1. Coordinador de la carrera de Ingeniería Civil y catedrático del Jepartamento de Mecánica Estructural de la UCA.

2. Catedrático del Departamento de Mecánica Estructural de la UCA. 


\section{Aspectos generales}

La sismología es la ciencia que estudia los terremotos, sus causas y sus efectos. Los terremotos son vibraciones de la tierra, causadas por el paso de ondas sísmicas irradiadas desde una fuente de energía. Dicha fuente es simplemente una falla geológica, la cual se puede definir como un plano de debilidad en la conteza terrestre (roca), que puede romperse, debido a fuerzas internas generadas, en última instancia, por las distribuciones de calor y temperalura, en el interior de la tierra. Este calor origina la dinámica interna de la tierra, causando el movimiento de diferentes placas tectónicas, que conforman la corteza lerrestre (una capa con un promedio de 30 kilómetros de espesor), las cuales flotan sobre el manto (una capa de 2500 kilómetros de espesor), el cual se encuentra en estado de fusión parcial o viscoso. La mayor concentración de actividad sísmica y de terremotos destructores del planeta se encuentra, precisamente, en los límites o bordes de estas placas tectónicas o fragmentos de corteza. Estos bordes se suelen encontrar sometidos a grandes tensiones, las cuales pueden dar lugar, de manera directa, a la generación de terremotos. Por otro lado, estas altas tensiones se asocian también a la existencia y concentración de fallas geológicas en las regiones cercanas a los bor-
Los terremotos son vibraciones de la tierra, causadas por el paso de ondas sísmicas irradiadas desde una fuente de energía. Dicha fuente es simplemente una falla geológica, la cual se puede definir como un plano de debilidad en la corteza terrestre mentales o de origen. El primero es la hora de origen, es decir, la hora en que comienza la ruptura de la falla geológica y no se refiere a la hora en que la sacudida se comienza a sentir en alguna localidad específica. El segundo parámetro es la localización del foco sísmico, que es el punto en la falla donde empieza la ruptura. Dicha localización comprende la determinación de la profundidad focal en kilómetros, medida desde la superficie terrestre, y de las coordenadas geográficas en latitud y longitud de la proyección del foco en la superficie, conocida como epicentro. El tercer parámetro es la magnitud, que es una medida del tamaño del terremolo, se mide en una escala continua y está asociada con la cantidad de energía liberada durante la ruptura. La magnitud es un valor único para cada terremoto y teóricamente no tiene un límite máximo, aunque físicamente está restringida por el tamaño que puede tener una falla geológica, pues a mayor área de ruplura, la energía liberada aumenta. Con frecuencia, el concepto de magnitud es confundido con la intensidad. Esta última es una medida cualitativa de los efectos que causa el terremoto en un sitio específico sobre la superficie de la tierra. La intensidad se mide en una escala discreta en números romanos y tiene un límite máximo. En El Salvador y el resto de América se ha utilizado la escala de des de placas. Estas fallas suelen ser muy activas, en cuanto a su capacidad para generar sismos.

En El Salvador y en otros países de América Latina suelen utilizarse, además de la palabra "tепеmoto", los términos "sismo" y "temblor". La palabra "terremoto" se refiere a un movimiento que ha causado grandes daños y muchas víctimas. El término "sismo" se utiliza para hacer referencia a una sacudida que no causa daños, pero que es muy sensible para la mayoría de personas, y la palabra "temblor" se utiliza cuando la sacudida es leve y no causa daño alguno. Estricta y técnicamente siempre y cuando exista una ruptura de falla, la palabra correcta es terremoto, con independencia de que el movimiento sentido en la superficie terreste sea leve o fuerte.

Para caracterizar a un terremoto en términos sismológicos, se necesitan tres parámetros funda-
Mercalli Modificada (MM), la cual va desde I (que es el caso de un temblor) hasta XII, cuando la destrucción es total. La intensidad, por lo tanto, será distinta de un punto a otro sobre la superficie terrestre, dependiendo de los efectos observados en objetos, infraestructura y terreno y de la percepción que han tenido las personas del fenómeno. Aunque la asignación de un grado de intensidad puede ser subjetiva, es de gran utilidad cuando no se tienen registros instrumentales del evento, como es el caso de los terremotos de los siglos XIX e incluso de los anteriores, ya que al estudiar los patrones de distribución de daños se puede estimar la magnitud y la localización del evento.

Los parámetros de origen (la hora, la localización y la profundidad) son obtenidos a través de aparatos conocidos como sismógrafos, cuyos registros son los sismogramas. Para fines de aplicación a la ingeniería, existe otro tipo de aparatos: 
los acelerógrafos, los cuales forman parte de los recursos de ingeniería sísmica de la UCA como se explica más adelante.

Hasta el momento, las técnicas para predecir los terremotos en los países desarrollados no han tenido éxito como tales y nadie es capaz de acertar la fecha, la hora, la localización ni el tamaño exacto de un evento sísmico. Por lo tanto, lo importante para la ingeniería es tratar de miligar el riesgo sísmico en futuros eventos, no importando si éstos ocurren a corto o mediano plazo, basándose en la historia sísmica de la región en estudio y en las lecciones aprendidas en terremotos destructores. La mitigación del riesgo sísmico debe ser, entonces, una tarea multidisciplinaria, donde técnicos en la materia, políticos, planificadores, urbanistas, constructores, etc., deben contribuir a que la provisión para la protección sea adecuada para disminuir de una manera razonable los daños que ocasionan los terremotos en la infraestructura de un pais. Hay que tener presente que aun en el supuesto de que se pudieran hacer predicciones exactas de la ocurrencia de un terremoto destructor, las medidas de evacuación de los habitantes reducirían el impacto únicamente en términos de vícti- mas mortales y heridos, pero si la infraestructura no es adecuada para resistir el evento sísmico, la desırucción y los daños materiales siempre producirán el mismo impacto económico y social.

\section{Tectónica y sismicidad}

El régimen tectónico de Centroamérica es el resultado de la interacción de cinco placas: la del Pacífico, Norteamérica, Cocos, Nazca y Sudamérica (Gráfica 1). La principal fuente de sismicidad en El Salvador es la zona de subducción, donde la trinchera o la fosa se encuentra a 125 kilómetros de la costa. Ahí, la placa de Cocos empieza a sumergirse por debajo de la placa del Caribe (Gráfica 2), alcanzando profundidades de hasta 300 kilómetros por debajo del istmo centroamericano (Dewey y Suárez, 1991; Burbach et al., 1984). Estos sismos son sentidos en todo el territorio nacional, porque se generan a grandes profundidades, aparte que pueden alcanzar grandes magnitudes. La magnitud máxima probable en esta zona de subducción es de $\mathbf{8 . 0}$

Otra importante fuente generadora de sismos es la que coincide con la ubicación de la cadena volcánica (Stoiber y Carr, 1977). La sismicidad de esta

\section{Gráfica 1}

Mapa de tectónica de placas para la región centroamericana (Weyl, 1980)

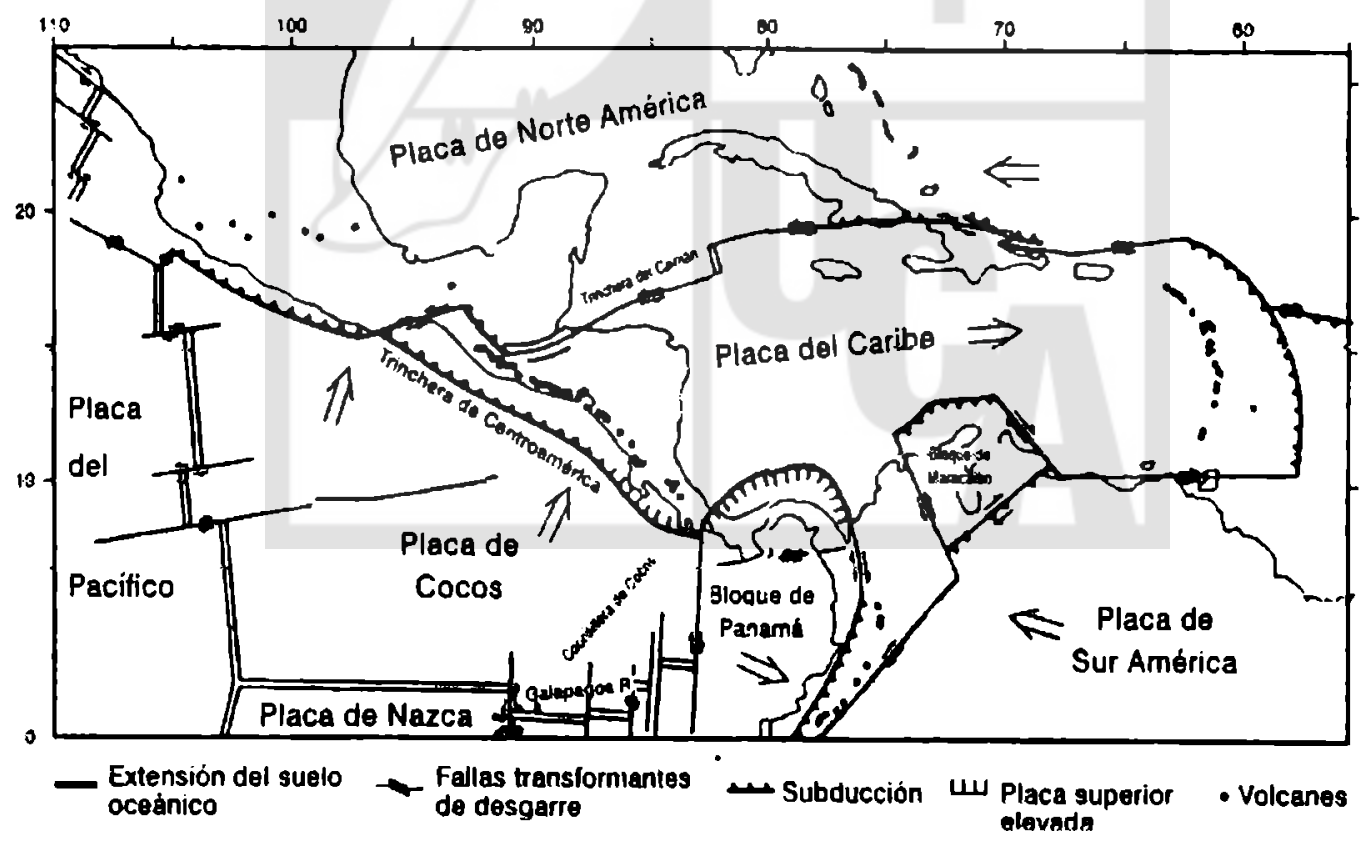




\section{Gráfica 2}

Corte transversal para la zona de subducción y eventos superficiales.

Los círculos son los focos de los terremotos y la línea punteada

la parte superior de la placa de Cocos (White y Harlow, 1993)

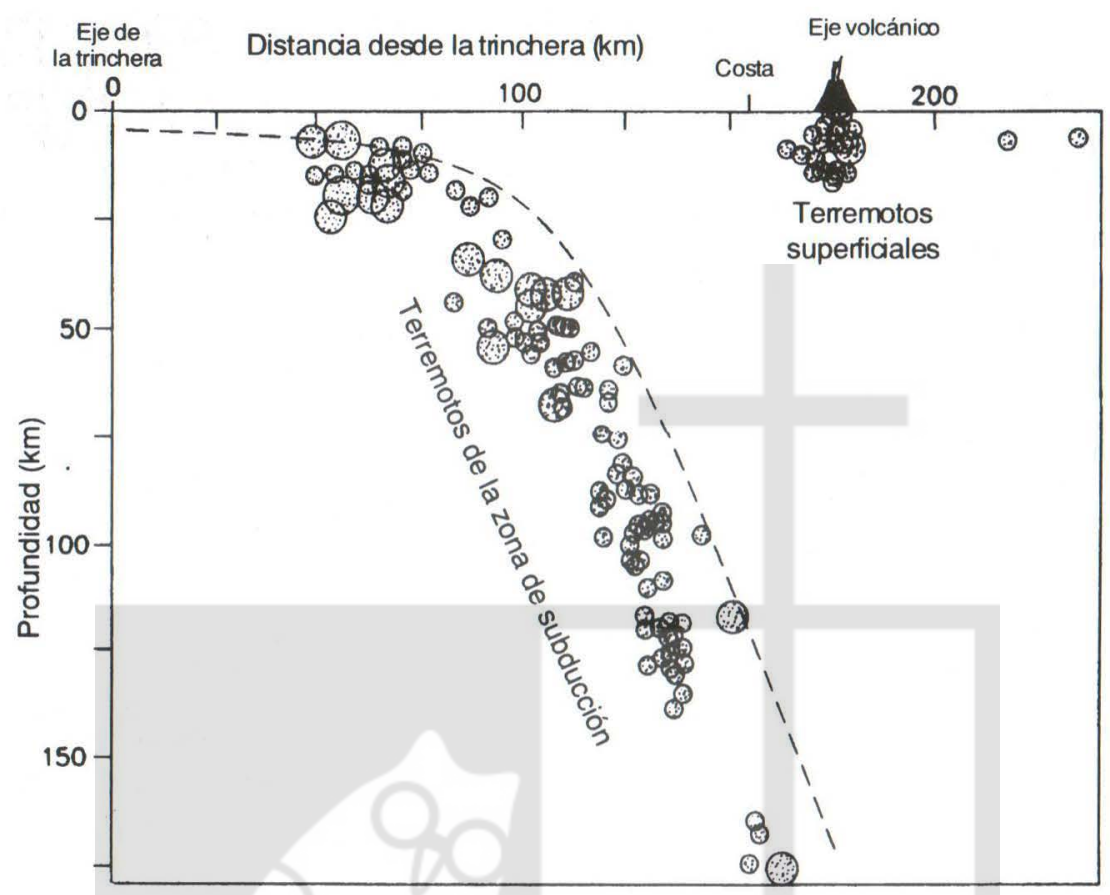

zona está concentrada en los primeros 25 kilómetros de espesor de la corteza y dentro de una franja paralela a la trinchera de unos 20 kilómetros de ancho. Aquí se encuentran los volcanes activos de la época del Cuatemario. Cabe señalar que cuando se afirma que un terremoto pertenece a la cadena volcánica, no necesariamente está asociado con actividad volcánica, sino que simplemente hace referencia a la región geográfica donde se origina. A diferencia de los eventos de la subducción, estos terremotos provocan daños en una área muy reducida, debido a que su foco es muy superficial, a pesar de que su magnitud es moderada. La magnitud máxima que se espera en esta zona es de alrededor de 6.7 .
Lo importante para la ingeniería es tratar de mitigar el riesgo sísmico [...] La mitigación del riesgo sísmico debe ser una tarea multidisciplinaria, donde técnicos en la materia, políticos, planificadores, urbanistas, constructores, etc. deben contribuir a que la provisión para la protección sea adecuada para disminuir de una manera razonable los daños que ocasionan los terremotos en la infraestructura de un país. 
có frente a las costas salvadoreñas, en las coordenadas geográficas $13.063^{\circ} \mathrm{N}$ (latitud norte) y $88.787^{\circ} \mathrm{W}$ (longitud oeste), y su foco tuvo una profundidad de 39 kilómetros. La magnitud se estimó en 7.7. Este evento pertenece a la zona de subducción.

Según el National Earthquake Infomation Center, el terremoto del 13 de febrero ocurrió a las 8:22:05 (hora local de El Salvador); su epicentro se localizó en las coordenadas $13.613^{\circ} \mathrm{N}$ (latitud norte) y $89.069^{\circ} \mathrm{W}$ (longitud oeste) dentro del territorio salvadoreño, al sur del lago de Ilopango; a una profundidad focal de 13 kilómetros y con una magnitud de 6.6. Este evento se originó en el sistema de fallas locales, ubicado en la cadena volcánica de El Salvador.

El 17 de febrero, a las 14:25 (hora local de El Salvador), ocurrió un sismo, cuyo epicentro está ubicado al sur del área metropolitana de San Salvador, en las coordenadas $13.660^{\circ} \mathrm{N}$ (latitud norte) y $89.248^{\circ} \mathrm{W}$ (longitud oeste). La profundidad focal fue de 5.1 kilómetros y su magnitud de 5.1 (Centro de Investigaciones Geotécnicas de El Salvador). Este evento pertenece también al sistema de fallas Jocales del pais.

Hasta el momento no se tiene información de estudios específicos sobre la intensidad de estos eventos, en la escala de Mercalli Modificada en los diferentes puntos de El Salvador. Es conveniente, por lo tanto, no asignar ni proporcionar intensidades hasta no tener una base de datos confiable para este propósito.

\section{La red acelerográfica digital de la UCA}

Una red acelerográfica es un conjunto de instrumentos ubicados generalmente sobre la superficie terrestre para registrar el movimiento del terreno durante un terremoto, en tres componentes (dos horizontales y una vertical) cerca de la fuente sísmica. Estos instrumentos reciben el nombre de acelerógrafos y el registro que proporcionan se denomina acelerograma —un gráfico de la aceleración del terreno, en función del tiempo, en cada una de sus tres componentes- La importancia de conocer los valores de aceleración del terreno durante un evento sismico radica en que los valores de fuerzas sísmicas que se imponen en las estructuras se relacionan y son proporcionales a dichas aceleraciones.

Esto implica que el conocimiento de las aceleraciones producidas por terremotos puede conducir a mejorar el conocimiento sobre los niveles de las fuerzas sísmicas, lo cual es necesario para el diseño adecuado de estructuras con un equilibrio óptimo entre seguridad y economía.

El personal de la universidad fue capacitado y ésta adquirió equipo para desarrollar el campo de la ingeniería sísmica como resultado de un proyecto en el cual, además de la UCA, participaron cinco instituciones europeas, entre 1995 y 1998 . Parte del equipo adquirido lo constituyeron diez acelerógrafos digitales. En febrero y principios de marzo de 1996, se instalaron nueve de ellos, ocho en dependencias del Ministerio de Salud Pública y Asistencia Social y uno en el Colegio Externado San José. En agosto del mismo año se instaló el último, en la Unidad de Salud de Tonacatepeque. En el Cuadro 1 se muestra la información registrada por estas diez estaciones acelerográficas de la UCA. En la Gráfica 3 se muestra un mapa con la distribución de la red. Los acelerogramas se obtienen en forma digital y contienen 200 valores de aceleración por cada segundo durante el evento. Estos solamente registran la sacudida si el valor de aceleración es superior al 0.6 por ciento de la aceleracion de la gravedad en la componente vertical o al 1 por ciento en cualesquiera de las componentes horizontales. Para mayor referencia sobre la red digital de la UCA puede consultarse el trabajo de Bommer et al. (1997) y Cepeda et al. (1997). Como se mencionó antes, los acelerogramas son de gran utilidad para la ingeniería, ya que son el punto de partida para analizar y diseñar cualquier estructura resistente a cargas sísmicas. 


\section{Cuadro 1 \\ Estaciones de la red acelerográfica digital de la UCA}

\begin{tabular}{|c|c|c|c|c|c|}
\hline Estación & Código & $\begin{array}{l}\text { Latitud } \\
\text { Norte } \\
\text { (grados) }\end{array}$ & $\begin{array}{l}\text { Longitud } \\
\text { Oesle } \\
\text { (grados) }\end{array}$ & $\begin{array}{c}\text { Condicioncs } \\
\text { de } \\
\text { silio }\end{array}$ & $\begin{array}{c}\text { Elcvación } \\
\text { (mclros sobre } \\
\text { el nivel del } \\
\text { mar) }\end{array}$ \\
\hline 1. Unidad de Salud de Armenia, Sonsonate & UARM & 13.744 & 89.501 & Suelo & 570 \\
\hline 2. Hospital San Bartolo, San Salvador & CSBR & 13.704 & 89.106 & Suelo & 622 \\
\hline 3. Unidad de Salud de La Libertad & ULLB & 13.486 & 89.327 & Aluvión & 16 \\
\hline 4. Unidad de Salud de San Pedro Nonualco, La Paz & USPN & 13.602 & 88.927 & $\begin{array}{l}\text { Suclo, cresta } \\
\text { dorsal este-ocsic }\end{array}$ & 658 \\
\hline 5. Unidad de Salud de Panchimalco, San Salvador & UPAN & 13.614 & 89.179 & $\begin{array}{l}\text { Roca, fondo } \\
\text { valle estrecho, } \\
\text { eje nortc-sur }\end{array}$ & 613 \\
\hline 6. Hospital San Rafael, Santa Tecla & HSRF & 13.671 & 89.279 & Suelo & 912 \\
\hline 7. Unidad de Salud de Tonacalepeque, San Salvador & UTON & 13.778 & 89.114 & Suclo & 607 \\
\hline 8. Hospital Santa Gertrudis, San Vicente & HSGT & 13.642 & 88.784 & Suclo & 373 \\
\hline 9. Colegio Externado San José, San Salvador & ESJO & 13.707 & 89.207 & Suelo & 675 \\
\hline 10. Hospital Santa Teresa, Zacalecoluca & HSTR & 13.517 & 88.869 & Suelo & 253 \\
\hline
\end{tabular}

El objetivo de la red es cubrir la actividad sísmica de la cadena volcánica de El Salvador y de la zona de subducción, cuyos terremotos, históricamente, han causado daños en distintas ciudades del país (Gráficas 4 y 5). El diseño de la distribución de esta red digital está basado sobre todo en la localización de tres fuentes de actividad sísmica importantes, en la cadena volcánica central y occidental del país: la zona delimitada por San Salvador y el lago de Ilopango, el área sureste del lago de Coatepeque al oeste de San Salvador y San Vicente, al este de San Salvador.

\section{Gráfica 3}

Distribución de la red Acelerográfica de la UCA

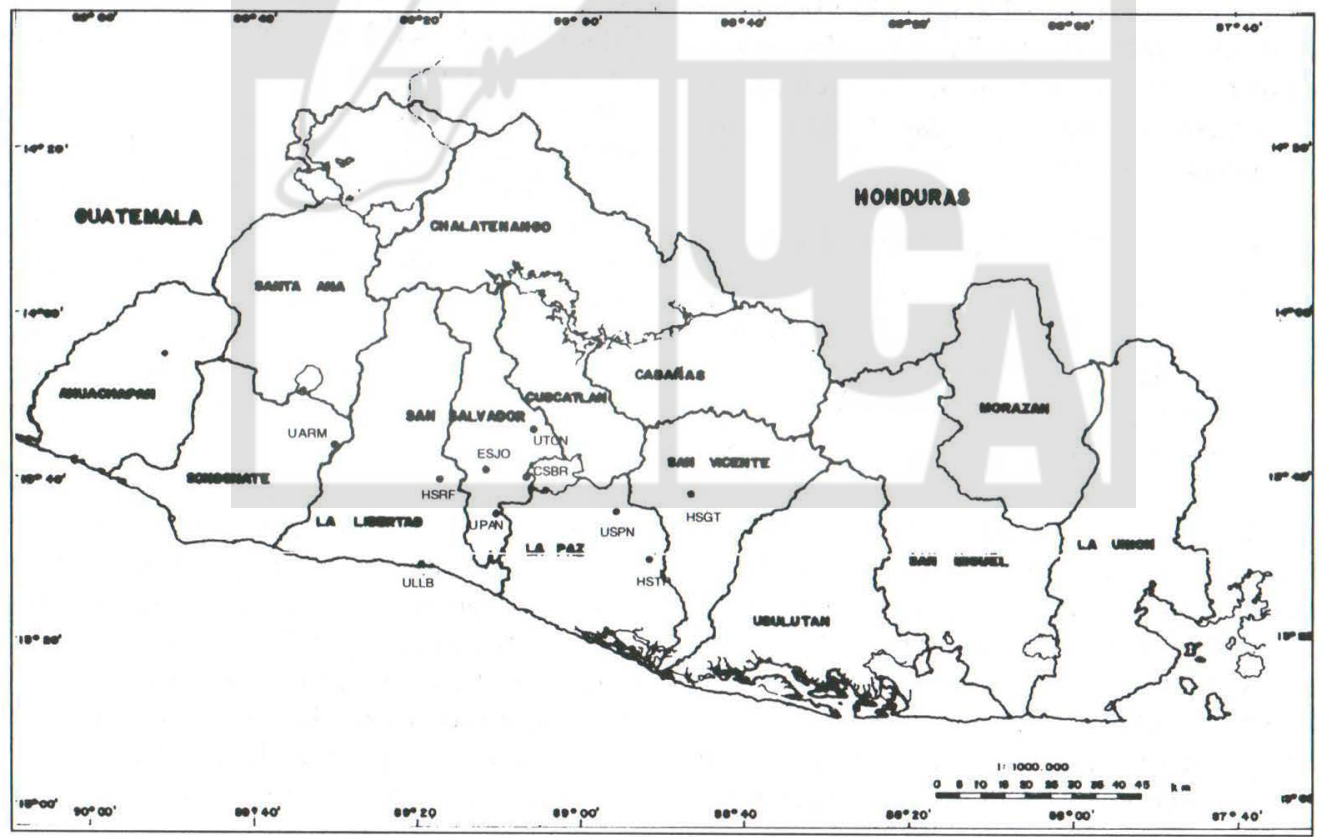




\section{Gráfica 4}

Mapa para contornos de isosistas con intensidad

Mercalli Modificada MM mayor a VII para terremotos de carácter superficial

(A) 1700-1799 (B) 1800-1899 (C) 1900-1990 (Harlow et al., 1993)
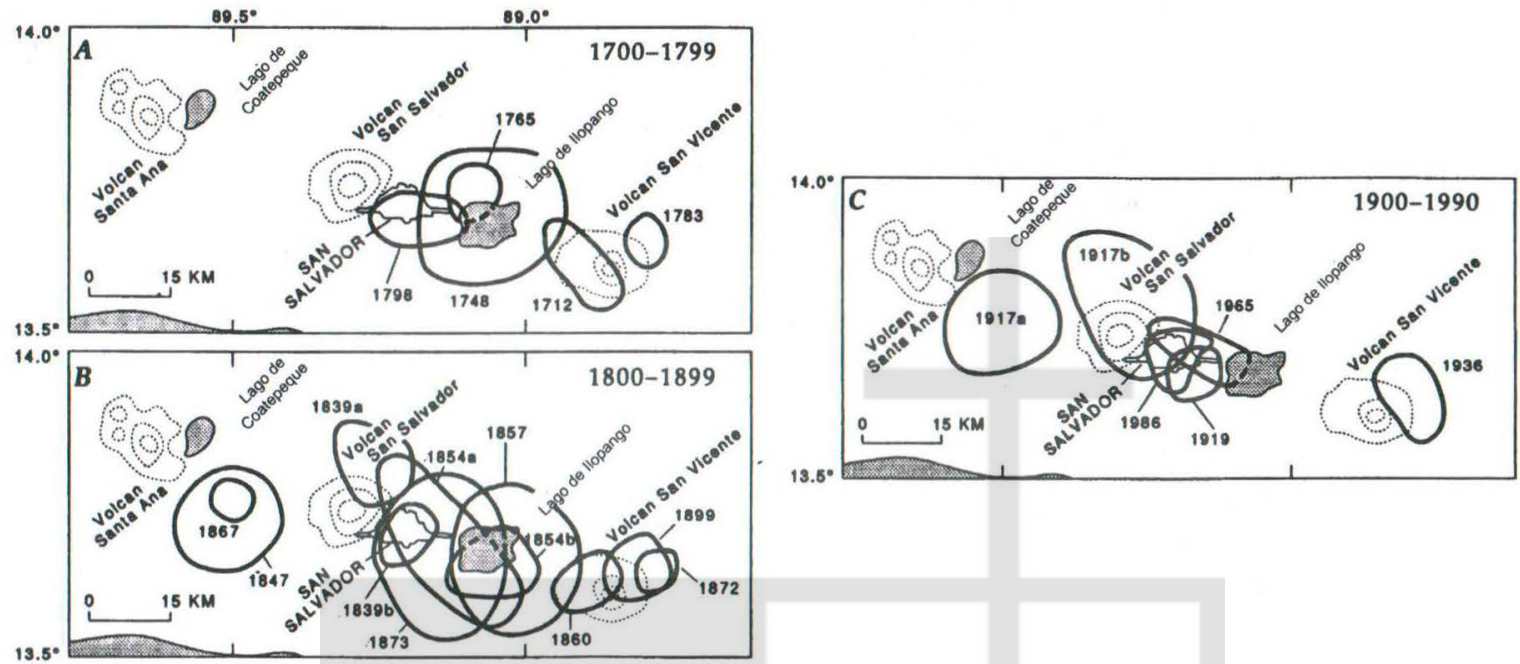

Gráfica 5

Mapa de isosistas para el terremoto del 19 de junio de 1982 en la zona de subducción Las intensidades corresponden a la Escala Mercalli Modificada MM (Álvarez, 1982)

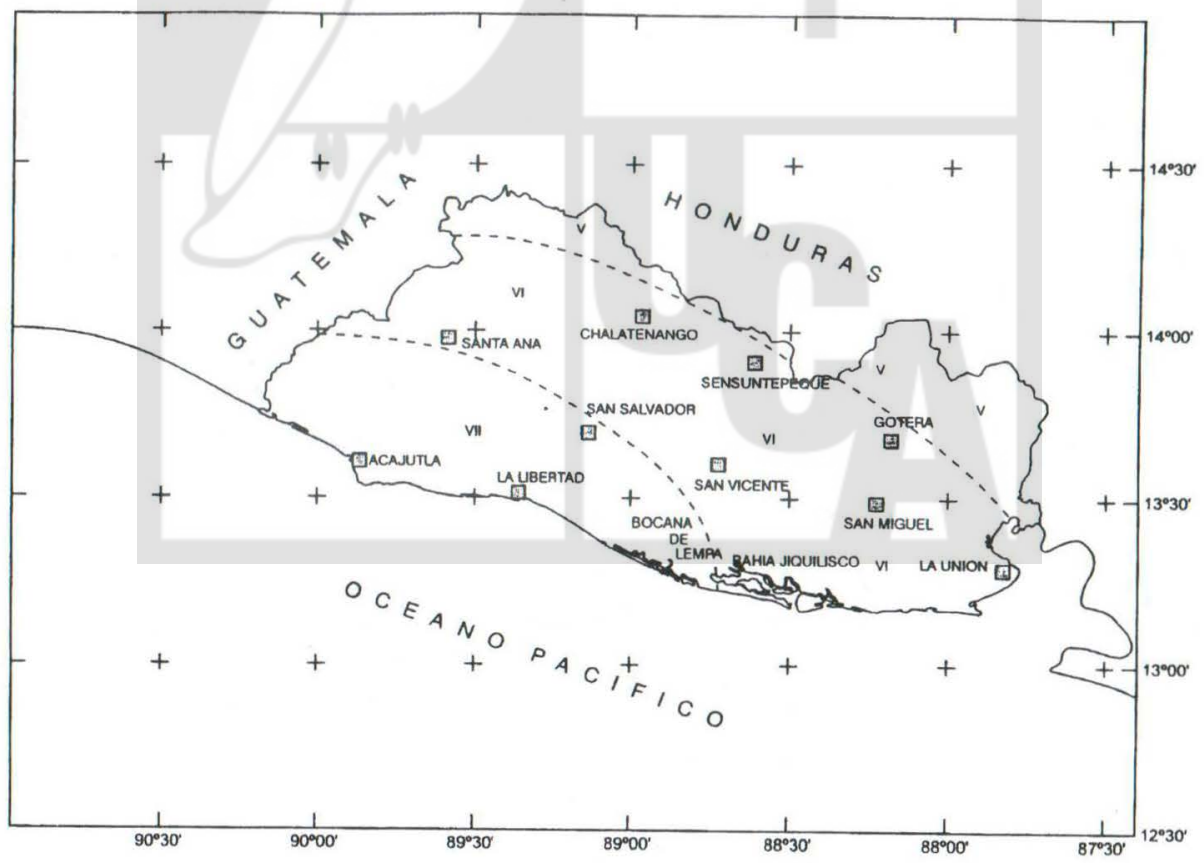


Los terremolos ocurridos a comienzos de este año pertenecen a la subducción y a la cadena volcánica salvadoreña. Según las evaluaciones de sismicidad que la UCA ha efectuado durante estos años (Salazar et al., 1997), terremotos con características comparables al del 13 de enero tenderían a repetirse en ciclos de setenta años. Esto pone de manifiesto la importancia de los registros obtenidos durante este terremoto. Hasta el momento, es el primer terremoto de subducción cuya magnitud es superior a 7 y que fue monitoreado por las estaciones acelerográficas de la UCA. Al mismo tiem- po, los terremotos del 13 y el 17 de febrero, originados en los sistemas de fallas locales de la cadena volcánica y cuyo ciclo de ocurrencia oscila entre los quince y veinte años, son los primeros en registrarse a distancias cortas y medianas del epicentro, en la historia sísmica del país.

\section{Resultados obtenidos}

En las Cuadros 2, 3 y 4 se muestran las aceleraciones y las velocidades máximas lcídas de los registros obtenidos en los terremotos del 13 de enero, y del 13 y 17 de febrero, respectivamente.

\section{Cuadro 2}

Aceleraciones y velocidades máximas del terremoto del 13 de enero

\begin{tabular}{|c|c|c|c|c|c|c|}
\hline \multirow[t]{2}{*}{ Estación } & \multicolumn{3}{|c|}{ Aceleración máxima $\left(\mathrm{cm} / \mathrm{s}^{2}\right)$} & \multicolumn{3}{|c|}{ Velocidad máxima $(\mathrm{cm} / \mathrm{s})$} \\
\hline & Norte-sur & Vertical & Este-oeste & Norte-sur & Vertical & Este-oeste \\
\hline UARM & 594.20 & 218.80 & 445.70 & 49.65 & 19.77 & 53.18 \\
\hline CSBR & 153.60 & 162.20 & 194.70 & 25.21 & 14.74 & 31.32 \\
\hline ULLB & 1108.00 & 624.30 & 563.80 & 54.91 & 16.51 & 35.86 \\
\hline USPN & 564.10 & 433.10 & 480.50 & 37.40 & 18.57 & 24.94 \\
\hline UPAN & 173.60 & 86.42 & 151.20 & 9.382 & 8.24 & 9.25 \\
\hline HSRF & 484.10 & 240.40 & 476.40 & 57.03 & 18.12 & 33.84 \\
\hline UTON & 229.80 & 201.20 & 270.90 & 23.25 & 9.92 & 23.37 \\
\hline HSGT & -.- &.- & -.- & -.- & -.- & -.- \\
\hline ESJO & 295.50 & 149.90 & 272.50 & 25.36 & 12.35 & 17.22 \\
\hline HSTR & 254.90 & 248.50 & 302.30 & 12.26 & 11.43 & 22.40 \\
\hline
\end{tabular}

Cuadro 3

Aceleraciones y velocidades máximas del terremoto del 13 de febrero

\begin{tabular}{lrrrrrr}
\hline \multirow{2}{*}{ Estación } & \multicolumn{3}{c}{ Aceleración máxima $\left(\mathrm{cm} / \mathrm{s}^{2}\right)$} & \multicolumn{3}{c}{ Velocidad máxima $(\mathrm{cm} / \mathrm{s})$} \\
& Norte-sur & Vertical & Este-oeste & Norte-sur & Vertical & Este-oeste \\
\hline UARM & 28.24 & 25.77 & 36.05 & 4.20 & 1.35 & 2.34 \\
CSBR & 104.30 & 120.70 & 138.80 & 25.94 & 6.93 & 22.39 \\
ULLB & 89.30 & 35.76 & 91.60 & 4.62 & 3.13 & 4.50 \\
USPN &.-- &.-- &.-- &.-- &.-- &.-- \\
UPAN & 180.90 & 43.59 & 104.20 & 9.58 & 2.07 & 4.78 \\
HSRF & 46.79 & 22.33 & 39.65 & 6.73 & 2.01 & 4.82 \\
UTON & 247.10 & 235.60 & 338.30 & 24.93 & 10.54 & 30.19 \\
HSGT & 425.40 & 228.90 & 233.00 & 14.67 & 4.04 & 6.15 \\
ESJO & 121.30 & 51.07 & 97.16 & 18.57 & 2.78 & 6.62 \\
HSTR & 400.20 & 256.10 & 296.80 & 20.11 & 9.65 & 20.19 \\
& & & & & & \\
\hline
\end{tabular}




\section{Cuadro 4}

Aceleraciones y velocidades máximas del terremoto del 17 de febrero

\begin{tabular}{lrrrrrr}
\hline \multirow{2}{*}{ Estación } & \multicolumn{3}{c}{ Aceleración máxima $\left(\mathrm{cm} / \mathrm{s}^{2}\right)$} & \multicolumn{3}{c}{ Velocidad Máxima $(\mathrm{cm} / \mathrm{s})$} \\
& Norte-sur & Vertical & Este-oeste & Norte-sur & Vertical & Este-oeste \\
\hline UARM & 26.56 & 19.63 & 17.18 & 1.55 & 0.71 & 0.77 \\
CSBR & 59.17 & 26.89 & 68.17 & 4.17 & 1.54 & 3.39 \\
ULLB & 25.37 & 7.76 & 14.30 & 0.77 & 0.24 & 0.78 \\
USPN &.-- &..-- &.-- &.-- &.-- &.-- \\
UPAN & 120.9 & 39.88 & 43.33 & 2.11 & 0.86 & 1.06 \\
HSRF & 81.15 & 99.11 & 82.25 & 3.10 & 2.19 & 4.44 \\
UTON & 32.60 & 27.01 & 46.36 & 1.73 & 0.74 & 1.54 \\
HSGT &.-- &..-- &.-- &.-- &.-- &.-- \\
ESJO & 92.48 & 71.54 & 78.53 & 2.57 & 1.75 & 3.73 \\
HSTR &.-- &.-- &.-- &.-- &.-- &.-- \\
\hline
\end{tabular}

El Cuadro 5 muestra las distancias epicentrales de las estaciones que registraron cada uno de los eventos presentados. El concepto de distancia epicentral se define como la distancia entre un punto y el epicentro de un evento sísmico.

\section{Cuadro 5}

Distancias epicentrales (kilómetros) por estación y evento sísmico

\begin{tabular}{lccc}
\hline Estación & 13 de enero & 13 de febrero & 17 de febrero \\
\hline UARM & 108.0 & 48.8 & 28.8 \\
CSBR & 79.1 & 10.9 & 16.1 \\
ULLB & 74.9 & 31.2 & 21.1 \\
USPN & 61.7 &.-- &.-- \\
UPAN & 74.4 & 11.9 & 9.0 \\
HSRF & 85.9 & 23.5 & 3.6 \\
UTON & 86.9 & 18.9 & 19.5 \\
HSGT &.-- & 30.9 &.-- \\
ESJO & 84.7 & 18.2 & 6.8 \\
HSTR & 51.2 & 24.1 &.-- \\
\hline
\end{tabular}

Las Gráficas 6, 7 y 8 muestran la aceleración versus tiempo para las componentes norte-sur de los eventos del 13 de enero y del 13 y 17 de febrero, respectivamente. La escala de aceleración de los registros mostrados dentro de cada figura es la misma. Además, se han ordenado las gráficas de menor a mayor distancia epicentral.

\section{Análisis de resultados}

La intensidad de la sacudida producida por un evento sísmico en una localidad depende sobre todo de tres aspectos: la magnitud del sismo, la distancia respecto al origen del mismo y las condiciones geológicas y topográficas del sitio donde el evento es percibido. La Gráfica 9 muestra las componentes norte-sur, registradas en San Salvador, durante los tres eventos tratados. De los tres aspectos anteriores, el correspondiente a las condiciones del sitio es constante en los tres registros. Nótese la importancia de la magnitud del sismo, tanto en la amplitud del movimiento como en su duración. La relación es directamente proporcional. A mayor magnitud, la amplitud y duración de la sacudida aumenta. Esta comparación, sin embargo, es limitada porque 
los registros corresponden a diferentes distancias epicentrales, es decir, varían dos de los tres aspectos indicados antes.

La dependencia de la intensidad de la sacudida respecto a la magnitud es más clara en la Gráfica 10 , donde se muestran las componentes norte-sur, registradas en Tonacatepeque, durante los eventos del 13 y del 17 de febrero. En este caso, las distancias epicentrales son casi iguales, sólo con 0.6 kilómetros o 600 metros de diferencia. Las condiciones del sitio son iguales, pues se trata de una misma estación. Las magnitudes, sin embargo, son diferentes. Aquí se evidencia la enorme influencia de la magnilud, tanto en la amplitud como en la duración de la sacudida, al comparar ambos registros.

\section{Gráfica 6}

Terremoto del 13 de enero del 2001. Trazas de aceleración versus tiempo de componentes norte-sur ordenadas según su distancia epicentral

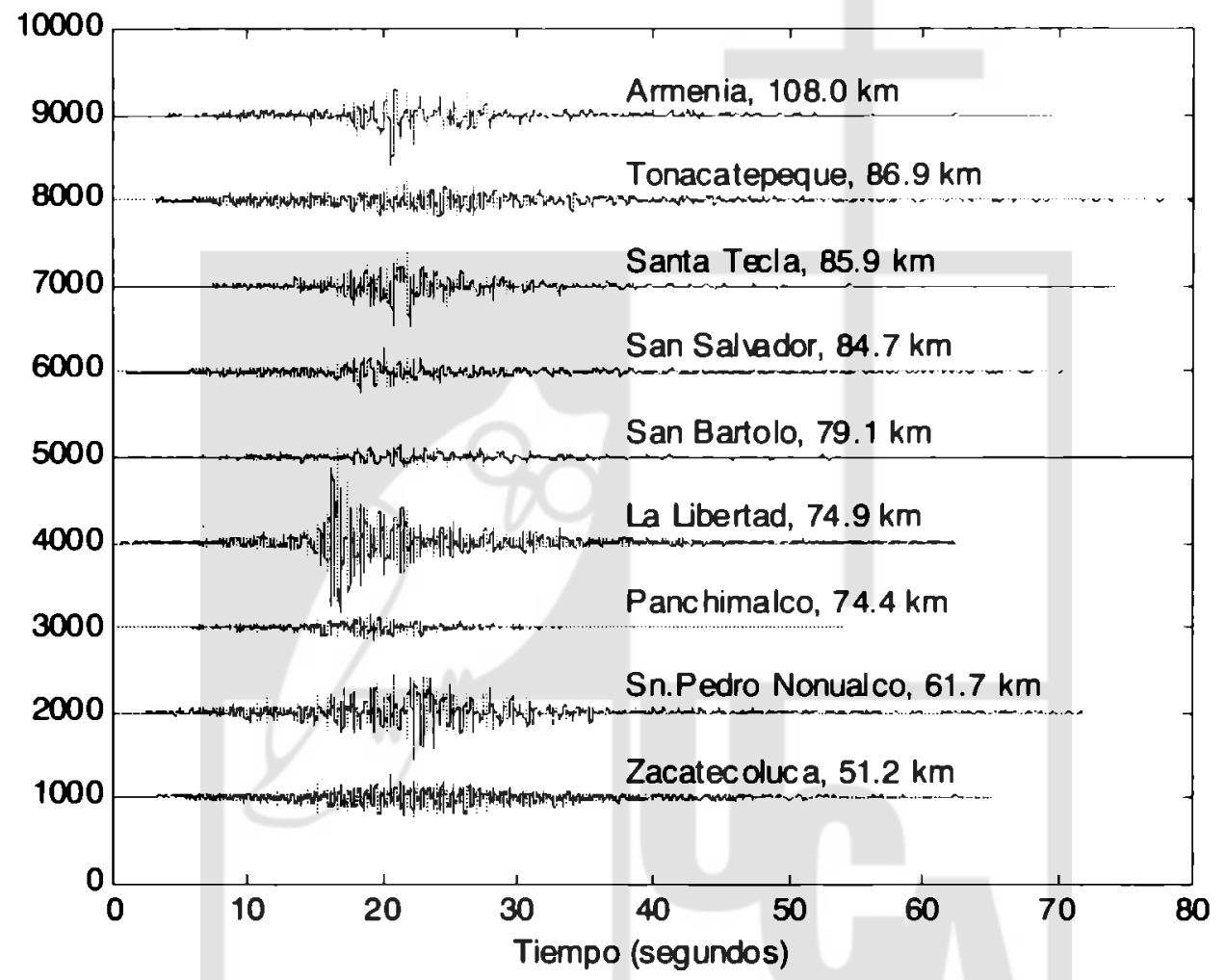

Para revisar la relación entre la distancia epicentral y la intensidad de la sacudida, se hará referencia a la Gráfica 11 . La caracterización de la sacudida se ha hecho utilizando como parámetro el cociente entre la intensidad de Arias (Arias, 1970) y la duración definida por Trifunac y Brady (1975). Este parámetro puede ser definido como una intensidad promedio, pero referida a períodos de tiempo distintos. Mientras la inten- sidad de Arias se calcula para el registro completo, la duración se evalúa como el tiempo transcurrido entre el 5 y el 95 por ciento de la intensidad de Arias total. La Gráfica 11 muestra un análisis de los registros del sismo del 17 de febrero. Se observa con claridad que la intensidad promedio se reduce al aumentar la distancia epicentral. Se han calculado las intensidades promedio, considerando las tres componentes del movimiento. 


\section{Gráfica 7}

Terremoto del 13 de febrero del 2001 . Trazas de aceleración versus tiempo de componentes norte-sur ordenadas según su distancia epicentral

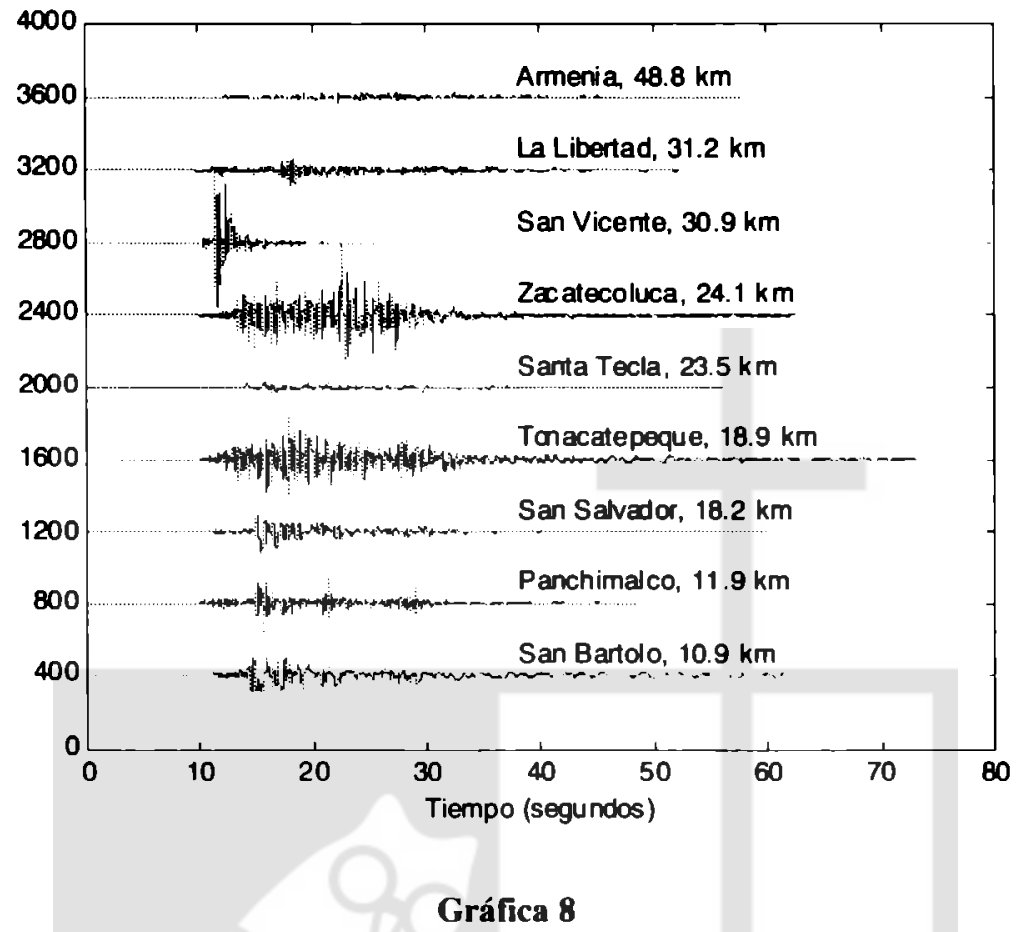

Sismo del 17 de febrero del 2001. Trazas de acelaración versus tiempo de componentes norte-sur ordenadas según su distancia epicentral

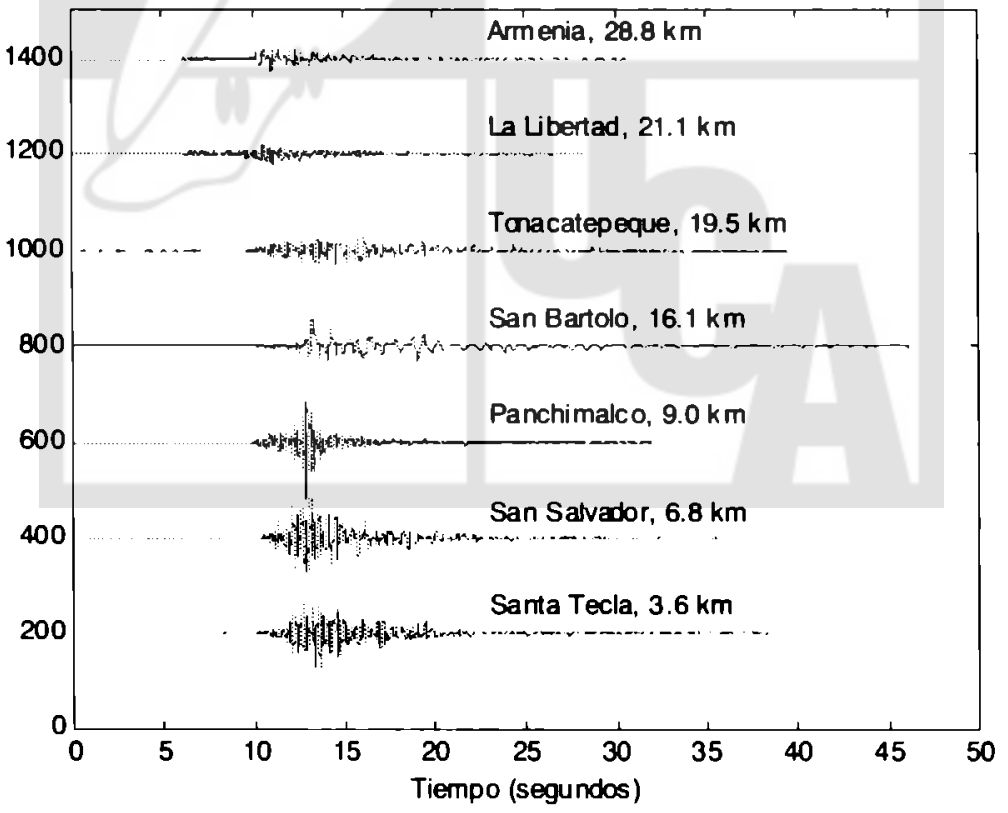




\begin{abstract}
Gráfica 9
Estación “Colegio Externado San José”, San Salvador. Trazas de aceleración versus tiempo de componentes norte-sur para los tres sismos principales del período enero-febrero del 2001
\end{abstract}

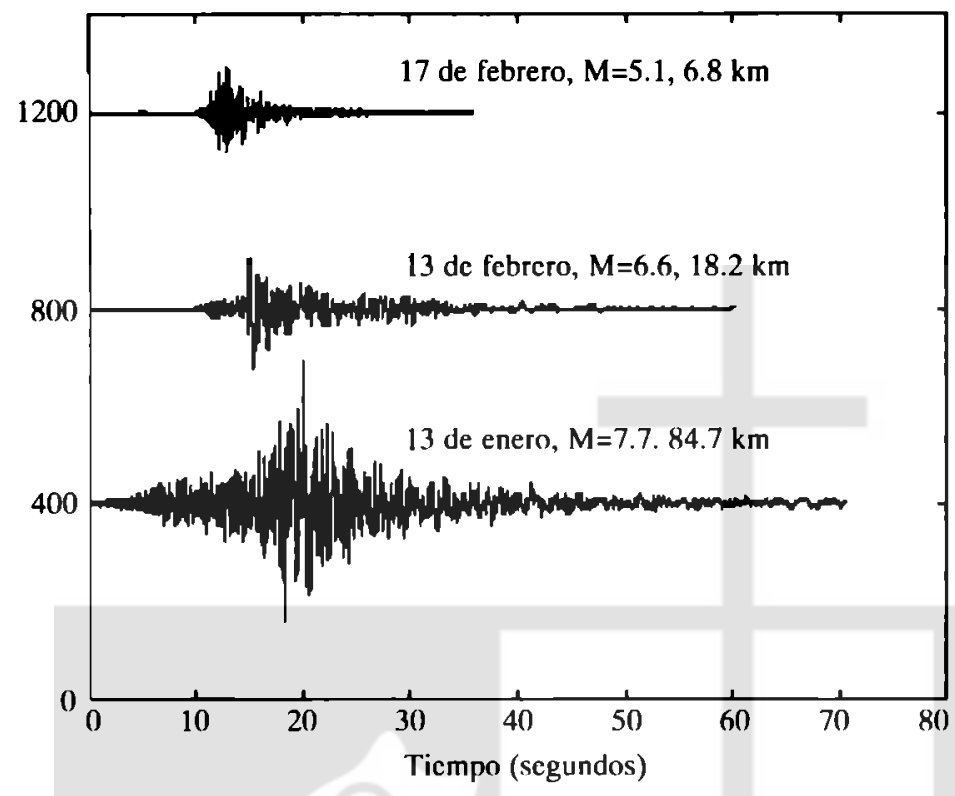

Los registros pueden, además, servir como indicación de las condiciones del sitio, en cuanto a la presencia de depósitos de suelos. Seed e Idriss (1982) establecieron valores promedios representativos de las características de los sitios según su geología. Los valores definidos corresponden al período predominante. El período representa, en este caso, el tiempo que toma a una capa de suelo realizar un ciclo completo de oscilación, en un movimiento horizontal. Como analogía, el período de un péndulo es el tiempo que le toma a éste realizar una oscilación completa. Los valores sugeridos por Seed e Idriss (1982) se muestran en el Cuadro 6.

\title{
Cuadro 6
}

Periodos promedio, representativos de diferentes condiciones de sitio

\begin{tabular}{cc}
\hline Condiciones de sitio & Período (segundos) \\
\hline Roca & 0.35 \\
Suelos duros con espesores menores a $60 \mathrm{~m}$ & 0.70 \\
Suelos duros con espesores mayores a $60 \mathrm{~m}$ & 0.87 \\
\hline
\end{tabular}

Esto indica que a mayor espesor de los depósitos del suelo, el período característico del sitio aumentará y el incremento será mayor en depósitos de suelos más blandos.
Con los valores de velocidad y aceleración máxima de los registros es posible hacer una estimación del período predominante del sitio donde está instalado un instrumento. Utilizando los valo- 
res correspondientes a las componentes norte-sur del Cuadro 2 se obtienen los valores de período mostrados en el Cuadro 7.

\section{Cuadro 7}

Períodos estimados en algunas estaciones de la red acelerográfica de la UCA

\begin{tabular}{lc} 
Estación & Período estimado (segundos) \\
\hline UPAN (Panchimalco) & 0.34 \\
ESJO & \\
(Colegio Externado, & \\
San Salvador) & 0.54 \\
CSBR (San Bartolo) & 1.03 \\
\hline
\end{tabular}

Estos valores estimados evidencian dos puntos principales. Primero, las condiciones de Panchimalco, a diferencia de San Salvador, corresponden a las de un lecho de roca superficial. Segundo, los espesores de depósitos del suelo son mayores en la zona de San Bartolo respecto a los del Colegio Externado, lo cual es consistente con perfiles litológicos realizados por diversos estudios en el área metropolitana de San Salvador, que muestran un aumento gradual en los espesores de suelos volcánicos desde el poniente (Colegio Externado) hacia el oriente (San Bartolo). Se supone que dichos materiales fueron depositados ahi por erupciones del antiguo volcán de llopango.

\section{Gráfica 10}

Estación Unidad de Salud Tonacatepeque. Trazas de aceleración versus tiempo de componentes norte-sur para los sismos del 13 y del 17 de febrero del 2001

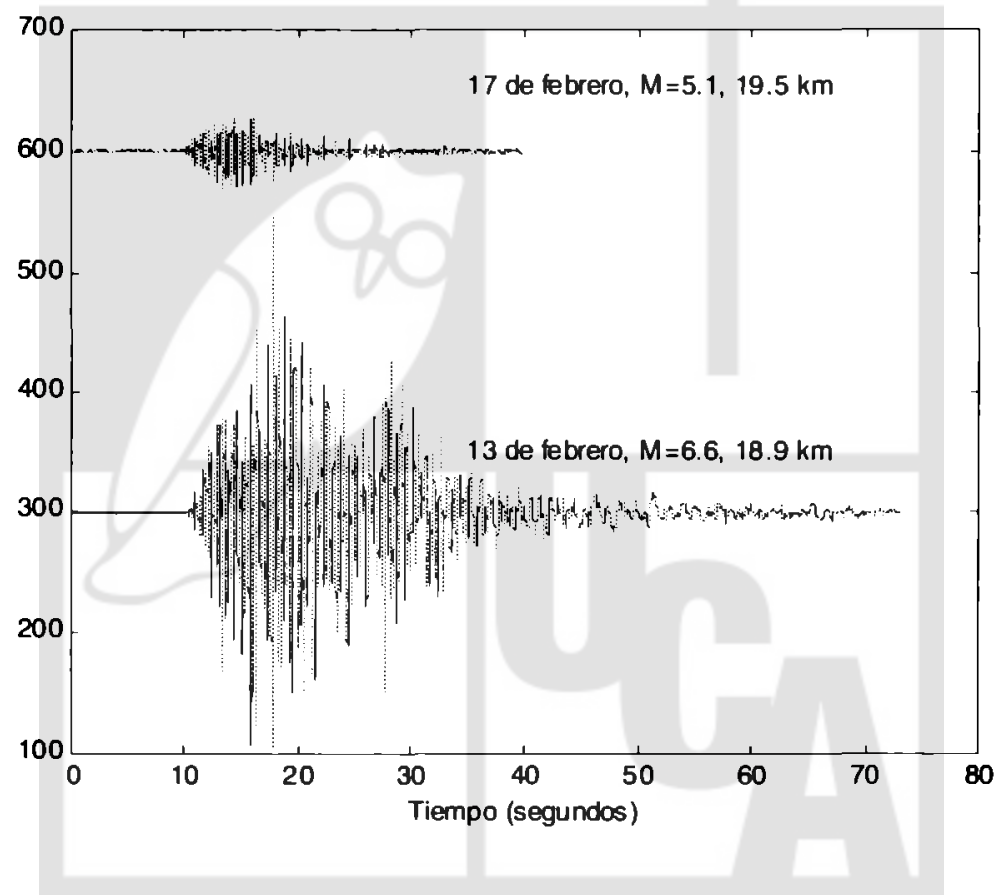

\section{Conclusiones}

Este análisis es muy breve y no permite obtener conclusiones definitivas sobre las características de los terremotos de los dos primeros meses del 2001. Con seguridad, a medida que se profundice en el estudio de la información recabada en este tiempo por otras instituciones y personas y por la UCA misma, las observaciones y conclusiones irán ganando mayor peso. Sin embargo, es posible adelantar algunos comentarios, a manera de conclusión, sobre lo que hasta ahora se ha podido ver en los datos disponibles. Conviene agrupar dichos comentarios según aspectos específicos. 


\section{Gráfica 12}

Intensidad promedio para registros de los sismos de 1982, 1986 y 2001. Círculos grandes: registros de zonas con daños considerables. Círculos pequeños: registros de zonas con daños menores o inexistentes. Nótese un umbral de separación cercano al valor de 0.2000 .

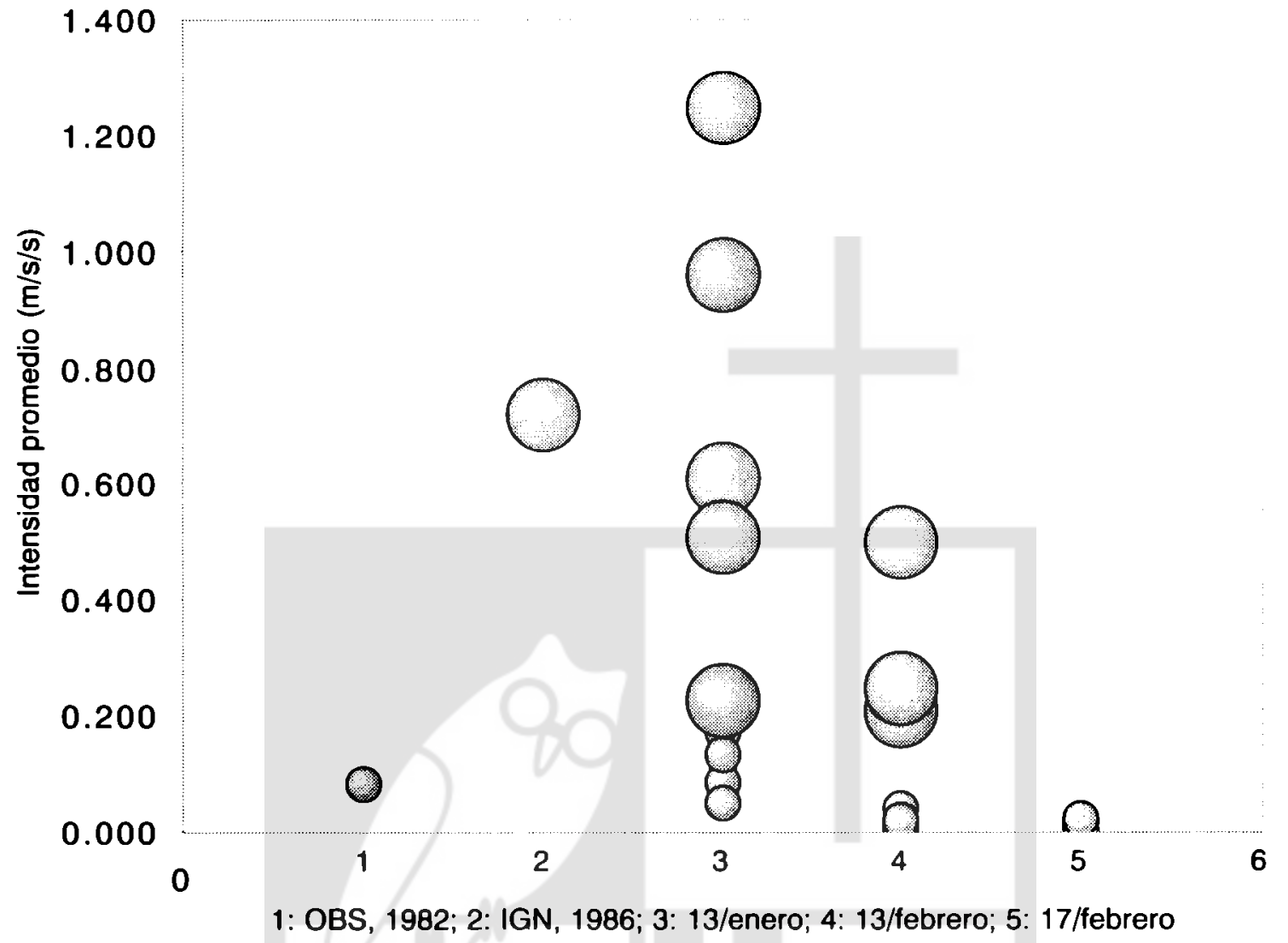

mención merecen el Imperial College de la London University, la Universidad Complutense de Madrid y el Instituto Geográfico Nacional de Madrid. Los aportes mayores los dieron el Dr. Julian Bommer de Imperial College, el Dr. Agustín Udias de la Universidad Complutense, el Dr. Julio Mezcua, entonces Director de Geofísica del Instituto Geográfico Nacional de Madrid, y el recordado Dr. Dimitri Papastamatiou, de la Universidad Técnica Nacional de Atenas.

El Ministerio de Salud Pública y Asistencia Social ofreció sus dependencias como albergues para la mayoría de los instrumentos y se reconoce que su aporte ha sido decisivo para el funcionamiento de la red. La Lic. Gloria de Calles, de la Unidad de Desastres, fue de mucha ayiida para fa- cilitar las gestiones. Los directores y empleados de los hospitales y de las unidades de salud han mostrado siempre su buena disposición para colaborar con el mantenimiento de los instrumentos. El Colegio Extemado San José, a través de sus rectores, desde el inicio de la red, Lic. Aníbal Meza, S. J. y Lic. Gerardo Guevara, y los empleados de la biblioteca han sido siempre muy diligentes en su colaboración.

A todos ellos, la UCA les agradece la confianza depositada y su aporte en el crecimiento del campo de la sismología y la ingeniería sísmica. La UCA espera que los resultados logrados en este período puedan beneficiar a la sociedad salvadoreña, al permitir conocer mejor el contexto sismológico del país. 
Los registros en forma digital y la información relacionada con ellos pueden obtenerse en forma gratuita en el sitio Web de la UCA: www.uca.edu.sv

\section{Bibliografía}

Álvarez, J. (1982). Informe técnico sobre aspectos sismológicos del terremoto en El Salvador, del 19 de junio de 1982. Centro de Investigaciones Geotécnicas, Ministerio de Obras Públicas, San Salvador.

Arias, A. (1970). "A Mcasure of Earthquake Inicnsity". En Seismic Design for Nuclear Power Plants, R. J. Hansen (cd.), Cambridge, Massachusctts, pp. 438-483.

Benito, B.; Cabañas, L. y Martínez, A. (1997). "Correlación entre parámetros de movimiento del suclo y daño estructural en El Salvador". Proceedings, Seminar on the Evaluation and Mitigation of the Seismic Risk in Central America Area, September 22-27, pp. 89-107.

Bommer, J. J.; Udías, A.; Ccpeda, J. M.; Hasbun, J. C.; Salazar, W. M.; Suárcz, A.; Ambrascys, N. N.; Buforn, E.; Cortina, J.; Madariaga, R.; Méndez, P.; Mczcua, J. y Papastamatiou, D. (1997). "A New Digital Accelerograph Network for El Salvador". Seismological Research Letters, Vol. 68, No. 3, pp. 426-437.

Burbach, G. Frohlic y Fumal, T. (1984). "Scismicity and Tectonics of the Subducted Cocos Plate". J. Geophys. Res., 89, B9, pp. 7719-7735.

Cepeda, J. M.; Salazar, W. M. y Bommer, J. J. (1997). "Caracterización de la red acelerográfica TALULIN".
Proceedings, Seminar on the Evaluation and Mitigation of the Seismic Risk in Central America Area, September 22-27, pp. 109-110.

Dewey, J y Suárez, A. (1991). "Seismotectonics of the Middle America". En Slemmons et al. (eds.), "Neotectonics of North America", Geol. Soc. Am. Decade Map, Vol 1, pp. 323-338.

Harlow, D.; White, R.; Rymer, M. y Alvarado, S. (1993). "The San Salvador Earthquake of 10 October 1986 and its Historical Context". Bull. Seism. Soc. America, 83, pp. 1143-1154.

Salazar, W. M.; Ambrascys, N. N. y Bommer, J. J. (1997). "Compilación de un calálogo sísmico para El Salvador y zonas aledañas". Proceedings, Seminar on the Evaluation and Mitigation of the Seismic Risk in Central America Area, September 22-27, pp. 67-76.

Sced, H. B. e Idriss, I. M. (1982). Ground Motions and Soil Liquefaction During Earthquakes. Earthquakc Enginecring Rescarch Inslitutc, Berkclcy, California.

Stoiber, R. y Carr, M. (1977). "Gcological Sctting Destructive Earthquakes in Central Amcrica". Geological Society of America Bulletin, Vol. 88, pp. $151-156$.

Trifunac, M. D. y Brady, A. G. (1975). "A Study of the Duration of Strong Earthquake Ground Motion". Bull. Seism. Soc. America, 65, pp. 581-626.

Wcyl, R. (1980). Geology of Central America. Gebruder Bortranger, Germany.

White, R. A. y Harlow, D. H. (1993). "Destructive UpperCrustal Earthquakes of Central America Since 1900". Bull. Seism. Soc. America, 83, pp. 1115-1142. 